\title{
BMJ Open Semistructured interviews regarding patients' perceptions of Choosing Wisely and shared decision-making: an Australian study
}

\author{
Jacqueline Allen, ${ }^{\oplus 1}$ Richard King, ${ }^{2}$ Stacy K Goergen,, ${ }^{3,4}$ Angela Melder, ${ }^{5}$ \\ Naama Neeman, ${ }^{6}$ Annemarie Hadley, ${ }^{2}$ Alison M Hutchinson ${ }^{7}$
}

To cite: Allen J, King R, Goergen SK, et al. Semistructured interviews regarding patients' perceptions of Choosing Wisely and shared decision-making: an Australian study. BMJ Open 2019;9:e031831. doi:10.1136/ bmjopen-2019-031831

\section{- Prepublication history and} additional material for this paper are available online. To view these files, please visit the journal (http://dx.doi.org/10. 1136/bmjopen-2019-031831).

Received 22 May 2019 Revised 26 July 2019 Accepted 29 July 2019
Check for updates

(C) Author(s) (or their employer(s)) 2019. Re-use permitted under CC BY-NC. No commercial re-use. See rights and permissions. Published by BMJ.

For numbered affiliations see end of article.

Correspondence to Dr Jacqueline Allen; jacqui.allen@monash.edu

\section{ABSTRACT}

Objectives This study aimed to examine how patients perceive shared decision-making regarding CT scan referral and use of the five Choosing Wisely questions with their general practitioner (GP).

Design This is a qualitative exploratory study using semistructured interviews.

Setting This study was conducted in a large metropolitan public healthcare organisation in urban Australia. Participants Following purposive sampling, 20 patients and 2 carers participated. Patient participants aged 18 years or older were eligible if they were attending the healthcare organisation for a CT scan and referred by their GP. Carers/family were eligible to participate when they were in the role of an unpaid carer and were aged 18 years or older. Participants were required to speak English sufficiently to provide informed consent. Participants with cognitive impairment were excluded.

Findings Eighteen interviews were conducted with the patient only. Two interviews were conducted with the patient and the patient's carer. Fourteen participants were female. Five themes resulted from the thematic analysis: (1) needing to know, (2) questioning doctors is not necessary, (3) discussing scans is not required, (4) uncertainty about questioning and (5) valuing the Choosing Wisely questions. Participants reported that they presented to their GP with a health problem that they needed to understand and address. Participants accepted their GPs decision to prescribe a CT scan to identify the nature of their problem. They reported ambivalence about engaging in shared decision-making with their doctor, although many participants reported valuing the Choosing Wisely questions.

Conclusions Shared decision-making is an important principle underpinning Choosing Wisely. Practice implementation requires understanding patients' motivations to engage in shared decision-making with a focus on attitudes, beliefs, knowledge and emotions. Systems-level support and education for healthcare practitioners in effective communication is important. However, this needs to emphasise communication with patients who have varying degrees of motivation to engage in shared decision-making and Choosing Wisely.

\section{INTRODUCTION}

Choosing Wisely is a de-implementation initiative aiming to reduce low value healthcare.
Strengths and limitations of this study

- The qualitative methods enabled a detailed examination of patients' attitudes and beliefs.

- Factors supporting the implementation of shared decision-making in Choosing Wisely were identified.

- Participants were referred for and attended a CT scan and nothing is known about use of the five Choosing Wisely questions among patients who were not referred for a CT scan.

Two main principles underpin Choosing Wisely: (1) the responsible stewardship of healthcare resources and (2) the inclusion of patients in healthcare decisions. ${ }^{1}$ Numerous studies have been conducted to assess the implementation of Choosing Wisely in relation to responsible stewardship. ${ }^{2-5}$ There is mixed research in relation to shared decision-making. Previous studies have identified that decision support tools facilitate shared decision-making. ${ }^{67}$ Other research has found that patients overestimate the benefits of medical interventions and underestimate the associated harms. ${ }^{8}$ However, few studies have been conducted about shared decision-making from the patient perspective in a de-implementation context such as Choosing Wisely.

\section{Choosing Wisely}

Initiated in 2012 by the American Board of Internal Medicine Foundation, the US-based Consumer Reports and nine US-based medical specialty societies, the Choosing Wisely campaign aims to avoid healthcare services, including tests and treatments, associated with evidence of low efficacy and/ or potential risk of harm to patients. ${ }^{4}$ Over 20 countries, including Canada, Italy, the UK and Australia, have joined the Choosing Wisely initiative. Choosing Wisely emphasises 


\section{Box 1 Five Choosing Wisely questions}

1. Do I really need this test or procedure?

2. What are the risks?

3. Are there simpler, safer options?

4. What happens if I don't do anything?

5. What are the costs? ${ }^{28}$

the responsibilities of medical professionals to justly distribute and manage healthcare resources. ${ }^{19}$ Additionally, Choosing Wisely emphasises shared decision-making between healthcare practitioners and patients. ${ }^{9}$

To date, studies investigating the effectiveness of Choosing Wisely implementation have addressed responsible stewardship in terms of the development of lists of tests and treatments to avoid, ${ }^{1011}$ impact studies,${ }^{1213}$ education interventions ${ }^{14}$ and physician attitudes. ${ }^{1516}$ Previous studies have identified a range of patient attitudes regarding Choosing Wisely. A Canadian study identified that patients endorsed Choosing Wisely values and de-implementing low value care. ${ }^{17}$ In an Australian evaluation, $61 \%$ of consumer participants indicated that they agreed with the Choosing Wisely campaign and the patient's role in reducing care of low value. ${ }^{18}$ However, $61 \%$ of participants expected that their medical practitioner should order all medical tests if they were unwell. ${ }^{18}$

\section{Shared decision-making}

Shared decision-making refers to the involvement of patients in making decisions about their health and healthcare with clinicians. ${ }^{6} 19$ The Choosing Wisely movement promotes shared decision-making between patients and healthcare professionals in relation to de-implementation of low value care. ${ }^{14}$ Shared decision-making is promoted through the five Choosing Wisely questions (listed in box 1) recommended for patients to use in discussions with their healthcare practitioner. ${ }^{18}$

Previous research about shared decision-making has largely focused on developing and testing decision support tools. ${ }^{19}$ In their systematic review, Stacey $e t$ $a t^{6}$ found good effectiveness of decision support tools on promoting patients' knowledge, communication between patients and practitioners, and patient satisfaction. Another systematic review found that medical practitioners endorse the use of decision support tools. ${ }^{20}$ Decision support tools have also been found to challenge practice because of lack of clinician time, lack of care continuity, lack of patient knowledge and power imbalance between patients and clinicians. ${ }^{71}$ The decision support tools investigated in these systematic reviews were based on high quality research evidence and addressed a range of focused health conditions. ${ }^{6719} 20$

Despite the substantial quantity of research in relation to decision support tools, ${ }^{6}$ limited research is available about shared decision-making and Choosing Wisely from the patient's perspective. Additionally, previous studies about shared decision-making have emphasised specified health problems. ${ }^{19}$ We explored patients' perspectives about shared decision-making in relation to CT scans and any medical condition with their general practitioner (GP) with regard to using the five Choosing Wisely questions. We selected CT scans for inclusion because reductions in CT scans for nominated conditions are one important target area of Choosing Wisely due to the risk of exposure to unnecessary radiation. ${ }^{19}$

\section{Research question}

How do patients perceive shared decision-making about CT scan referral and use of the five Choosing Wisely questions with their GP?

\section{METHODOLOGY}

The research design was qualitative exploratory using interviews. The Standards for Reporting Qualitative Research (SRQR) guidelines were used to report the study methodology.22

\section{Patient involvement in research}

Patients were not involved in the development of the research question, study design, recruitment or conduct of the study. However, the research was designed to elicit patients' perceptions.

\section{Conceptual framework}

The Theoretical Domains Framework (TDF) formed the conceptual framework underpinning this study. The TDF was selected because it was developed and validated from a synthesis of the 33 theories of behaviour change best suited to implementation research and practice. ${ }^{23-25} \mathrm{We}$ adopted the most recently published version of the TDF, which comprises 14 domains. The domains focus on individual motivation for behaviour and change including knowledge, beliefs, memory and decision processes, social and environmental influences, and emotion. ${ }^{25}$

\section{Setting}

The setting comprised a large metropolitan public healthcare network in south-eastern Australia. Following referral by their GP, patients attended the health network for an outpatient CT scan. The public healthcare network provides acute, subacute and outpatient services, including medical imaging, to a culturally and socioeconomically diverse community. Selection of this setting was expected to maximise variation and opportunities for information about patients from different cultural and socioeconomic backgrounds.

\section{Participants}

Purposive sampling using maximum variation for educational background and for socioeconomic status was used to select, for a semistructured interview, up to 20 patients plus or minus carers/family. All patients were aged 18 years or older. Participants were attending the healthcare organisation for a CT scan having been referred by their GP. Where carers/family accompanied the patient, and 
with the patient's permission, the carer/family member was invited to participate in the interview. Carers/family were eligible to participate when they were in the role of an unpaid carer, as nominated by the patient. Participants were required to be at least 18 years of age and speak English sufficiently to provide informed consent. Where participants had a diagnosis of dementia or cognitive impairment recorded on the GP referral, they were not approached to participate.

\section{Data collection tools and guidelines}

Data collection tools and guidelines comprised a demographic questionnaire and a semistructured interview guide. These tools and guidelines were developed using the TDF and an earlier unpublished literature review as guides. The demographic questionnaire included questions about the participant's age, gender, country of birth, presenting health problem, educational background and employment. Interview guidelines comprised questions regarding the participant's perceptions of shared decision-making with their GP in relation to their CT scan and their perceptions of the five Choosing Wisely patient questions. The interview guide for the study is presented in online supplementary file 1 .

\section{Procedure and data collection}

Reception staff in the imaging department at the participating healthcare organisation identified participants who met the selection criteria. With the participant's permission, the researcher (JA) used the Participant Information and Consent Form (PICF) to introduce the study including the overall goal of improved understanding of patients' perceptions about shared decision-making in order to support patients' conversations with their doctors. Patients who agreed to participate were invited to nominate their informal carer to participate as well. Following an explanation of the study guided by the PICF, written consent from patients and carers was obtained. All participants were provided with a copy of the PICF. The researcher then invited the patient, and if applicable their carer, to complete the demographic questionnaire to establish their eligibility to participate in the study. The researcher invited eligible patients and, if acceptable, their carer to participate in a face-to-face semistructured interview in a private office at the imaging department after their scan, or to participate, at a later date, in a telephone interview of no more than 45-min duration. With permission, the semistructured interview was audio-recorded for transcription. Interviews were conducted by the first author, a registered nurse with professional education in interviewing, at psychology Master's degree and nursing PhD level, and experience in interviewing patients and carers in both community health and research contexts. A professional transcriber transcribed the data.

\section{Data analysis}

Demographic data were entered into Statistical Package for the Social Sciences V.21. Categorical information was analysed using frequencies. The inductive analysis technique of thematic analysis was used to analyse interview data and to make decisions about data saturation. ${ }^{26} 27$ Data saturation occurs when similar codes and categories are identified across interviews with subsequent interviews identifying no new codes and categories. ${ }^{26}{ }^{27}$ As the number of participants required to achieve data saturation varies by research project, the research team decided to conduct an additional six interviews to confirm data saturation.

Guided by the research aim and TDF, thematic analysis involved the comparing and contrasting of codes and categories within and between interviews to identify themes and subthemes. ${ }^{26}{ }^{27}$ The first author conducted the data analysis. The last author cross-coded interview transcripts. The first and last authors discussed codes, categories and themes to test the interpretation of the data and support a coherent interpretation of the interviews. All data relevant to the study are included in the article or uploaded as supplementary information.

\section{Ethics approvals}

In accordance with the Australian National Health and Medical Research Council guidelines, the Ethics Committees at the healthcare organisation and Deakin University provided ethics approvals. Patients and carers were voluntary participants in the study. Following an explanation of the study, guided by the PICF, participants provided verbal and written consent. All data were de-identified. Project identifier numbers were allocated to all qualitative information.

\section{FINDINGS}

Twenty-two people agreed to participate. Eighteen interviews were conducted with the patient only. At two patients' requests, interviews were conducted with both the patient and the patient's carer who had accompanied them to the medical imaging department. Similar codes and categories were identified during analysis of the first 14 interviews. An additional six interviews were conducted with no new codes and categories emerging. Therefore, data saturation was considered to be achieved after 20 interviews. Most interviews (17) were conducted face-to-face at the healthcare organisation after the participant's scan. Three interviews were conducted by telephone 1 week after their scan.

Six participants were aged less than 50 years, nine participants were aged 50-69 years and seven participants were aged $70-89$ years. Fourteen participants were female and 16 spoke English at home. Participants experienced a range of health conditions including gastrointestinal problems, respiratory conditions, cardiovascular conditions, neuropathy and back pain. Other demographic information is presented in online supplementary file 2.

Five themes resulted from the thematic analysis:

1. Needing to know.

2. Questioning doctors is not necessary. 
3. Discussing scans is not required.

4. Uncertainty about questioning.

5. Valuing the Choosing Wisely questions.

\section{Needing to know}

All participants noted that they required a CT scan because they needed to know what was wrong with them. All participants commented that their need to know what was wrong with them was the main reason for booking an appointment with their GP. According to one carer:

I don't think there was too much to decide. She [patient] complained about the pain and the doctor wanted to do this [CT scan] to see what's going on there. She just wants to feel a bit better. She would probably have any procedure. (P2)

The scan was important to most participants in order to plan and prepare for treatment to resolve their health problem. Three participants noted that the scan was important for their peace of mind so that they could prepare for pain in the future and rule 'sinister' things out. Two participants considered that the scan was important to help remove their pain. One participant wanted to be sure that their infection was gone. Another participant wanted to be properly diagnosed to stop people doubting that he had back problems. One patient commented:

...peace of mind is the right thing, but I think it's [the CT scan] just to know what's going on so I can prepare myself. (P7)

\section{Questioning doctors is not necessary}

Participants explained their perspectives about communicating with their doctor in the theme 'Questioning doctors is not necessary'. Many participants commented on their belief that their doctors made the right decision by requesting a CT scan for the quickest assessment of their illness. According to these participants, they did not have a discussion with their doctor or ask questions, as this was not perceived to be necessary.

Our doctor, she's a doctor who doesn't want you to have unnecessary tests. We know that about her because she said that. So that when she recommends a test we tend to just think, yeah. (P6)

According to several participants, their doctor explained radiation and reassured them about the risk, therefore there was no need to ask questions. According to other participants, because they had a relationship with their doctor, there was no need for discussion:

I've been seeing her [the GP] for a while, I haven't had many CTs or x-rays done but I vaguely remember years ago her explaining radiation and not to be too stressed about it. This time "No" because we've got an established relationship so she doesn't really need to rehash. (P3)
Several participants commented that they trusted their doctor and their doctor's knowledge, and complied with their doctor's suggestions and decisions because they wished to feel better. Additionally, since they trusted their doctor's judgement and knowledge, they considered that asking questions about the decision to have a CT scan was not important or necessary. One patient commented:

All through life you have to have x-rays. In the long run, it might cause some of your cancers. I don't know. But I don't think it would change me. You see people have got to keep believing in their GP. If you lose the trust. ... I wouldn't even go to a GP. That defeats the purpose. (P20)

Most participants considered that people of older generations and also from some traditional family backgrounds and cultures might feel that it was disrespectful to ask their doctor any questions. Several participants commented that when a patient is in pain or very unwell they would not be well enough to ask questions.

Two participants noted that they needed to be their own advocate with their doctor and ensure that they were listened to. According to one participant, being her own advocate was important, as doctors could not know everything and could assume that patients wanted a quick answer to their problems:

I think you've got to be your own advocate. ... You have to stand up and speak for yourself, and listen. ... Because sometimes they [the doctors] don't know better. (P4)

\section{Discussing scans is not required}

In the third theme 'Discussing scans is not required', participants explained their perspectives about deciding to have a CT scan. Most participants commented that they did not want more discussion with their doctor, because they knew what the scan was for, understood their CT scan and experienced no anxiety. Thirteen participants noted that as they had undergone at least one CT scan in the past, they were familiar with CT scans and understood what to expect. Nine participants reported that they were satisfied with the explanation about the purpose of the CT scan they received from their doctor.

I understand most of what is going on a lot of the time anyway, so I don't really need to ask a lot of questions. I do ask when I need to but this wasn't a case that I needed to. (P13)

Two participants did not want to ask any questions because they perceived this would make them anxious. One participant did not ask questions because she did not want to know details about her diagnosis. Four participants commented that they asked questions of their doctor during the consultation to clarify the need for the scan.

According to most participants, discussion about the pros and cons of their scan was not desirable because they 
had already received adequate information from their GP about CT scans at previous consultations and they had acquired a good understanding of the risks and benefits. Two participants noted that their doctor explained the pros and cons of their scan to them, and nine participants commented that this was not explained to them. Three participants reported their belief that they needed to have the scan done and therefore did not consider that questions about the pros and cons of the scan were necessary. One participant reflected that he should have asked about the pros and cons of his scan; however, he understood that his scan was a straightforward CT scan.

Most participants reported that as far as they were aware there were no major disadvantages of having a CT scan and the most important thing was to find out what was wrong with their health. Several participants considered that CT scans were safe. Other participants noted some disadvantages of CT scans. Four participants noted that people should not be exposed to CT scans too often due to radiation although this risk was considered minimal. Several participants reported that their doctor had informed them of this risk. According to one patient:

Why would there be a disadvantage [of the CT scan]? ... I've had three CT scans in 12 months, so I don't find that over excessive. (P1)

\section{Uncertainty about questioning}

In the theme 'Uncertainty about questioning', many participants expressed uncertainty and hesitation about the usefulness of the five Choosing Wisely questions for themselves and for others in decision-making with their doctors. Eighteen participants commented that they had not seen the five Choosing Wisely questions before. Some participants reported their belief that patients should use the questions yet many patients do not ask their doctors questions. Several participants noted that the doctor would need to explain the five Choosing Wisely questions to patients in order for the questions to be used. Many participants commented that they did not need to ask their doctors the five Choosing Wisely questions due to the quality trusting relationship that they had with their doctor and their assumption that the doctor knew best. According to these participants, people without a trusting relationship with their doctor would need to ask questions. Other participants considered that patients would only be able to ask questions of their doctor if they had a trusting relationship where their doctor would accept a patient asking questions.

I think if you have a good relationship or have trust with your doctor, your GP, I guess you just would assume that she is going to decide the right thing for you. (P5)

It depends on the relationship that they have with their doctor. I think that if you don't really know your doctor and you don't trust your doctor then it [the five Choosing Wisely questions] potentially can help. (P7)

I think some people would [ask the five Choosing Wisely questions] and some people wouldn't. Some people are very switched on and want to know things and other people still have that, well the doctor knows best. I'll do what you tell me. (P10)

Three participants were undecided about whether the five Choosing Wisely questions would assist a patient to weigh benefits against risks. One participant noted that he had everything explained to him and he would not need to question his doctor. However, where patients did not have a relationship with their doctor, they may need to use the Choosing Wisely questions to weight up benefits against risks for themselves. One participant considered that patients would agree with the doctor and not use the questions. One participant reflected that patients might be too afraid of the answers from their doctor, in relation to risks, if they were to ask the five Choosing Wisely questions. Some people would prefer not to know and would not use the questions. According to one patient:

It's a scary thing to ask questions you really sometimes don't want to hear what they've [the doctor] got to say. (P9)

Six participants commented that they would not use the five Choosing Wisely questions, as they needed their scan in order to recover their health. Several other participants commented that they would never opt to do nothing and therefore they would not ask their doctor the Choosing Wisely question 'What happens if I don't do anything?' According to two participants, doctors may not want patients to ask them questions. Further, it was perceived that some patients may not want to ask their doctors questions since they may feel that questioning the doctor is too confrontational. One patient noted:

Do I really need this test or procedure? I think it's almost questioning the doctor. I think there is potential for some doctors to have their nose out of joint. (P7)

\section{Valuing the Choosing Wisely questions}

All participants were invited to discuss the value of the questions. However, only 14 participants commented. These participants expressed a range of views about the value of the questions. Several participants considered the questions were valuable because they guided patients to reflect on decisions with their doctor and to take greater responsibility for their healthcare decisions. One carer participant reported their belief that some people did have unnecessary tests and procedures and that there may be associated risks that were not considered. According to this carer, some people thought that an X-ray test was just like taking a photograph with no risks and therefore the Choosing Wisely questions could be valuable in prompting patients to consider risks with their doctors. Another participant further explained that during a 
consultation with a doctor, patients were more concerned with what they thought that they needed. According to this participant, the questions would prompt a patient to consider additional priorities such as are there safer or cheaper options. Two participants reported that the questions would be valuable because they would increase the information available to patients, which would be reassuring for patients in making decisions with their doctors. Another two participants noted that the questions would be valuable as a memory prompt for patients. According to one patient:

I think it [the five Choosing Wisely questions] might jolt a person's memory. It might engage the patient on a different level. You know outside their paradigm of thinking. So it's a bit like a safety map. (P18)

Some participants commented that the five Choosing Wisely questions were valuable because availability of the questions would give patients permission to ask questions of their doctors. According to one participant, some people believed that they were not permitted to ask their doctors questions and needed to know that asking doctors questions was acceptable. Several participants noted that the questions would need to be brought to patients' attention by doctors to signal to patients that it was acceptable to ask questions.

I think ... some patients need to have that so that they're given permission in their minds to ask those questions. (P6)

Some people just do what they're told. But if there was a set of questions then they would know to ask wouldn't they? (P21)

Additionally, participants commented on a range of facilitators and barriers to using the five Choosing Wisely questions. These are listed in online supplementary file 3. Several participants reported that they did not find the Choosing Wisely question regarding costs was valuable as they considered that it was not applicable to the publicly funded Australian health setting. Two participants asked if the Choosing Wisely question pertaining to costs reflected monetary costs or human costs.

\section{DISCUSSION}

Findings indicate that participants presented to their GP with a health problem that they needed to understand and address. Participants accepted their GPs decision to prescribe a CT scan to identify the nature of the problem. Participants reported ambivalence about using the five Choosing Wisely questions with their doctor; although, many participants reported valuing these questions. Few past studies have examined using the five Choosing Wisely questions from the patient perspective. ${ }^{17} 18$ Findings from the current study contribute knowledge about patients' use of questions in healthcare contexts of de-implementation of low value care.
Previous research about shared decision-making has found decision support tools with clearly articulated decision choices are effective. ${ }^{6} 1920$ Asking questions, such as use of the five Choosing Wisely questions, is one part of shared decision-making. ${ }^{19}$ However, in the current study, findings indicate that all participants perceived that they required a solution to their health problem in the form of an investigatory intervention. Although many participants understood the value of Choosing Wisely in terms of healthcare stewardship, when the decision was about their own health directly, they expected their doctor to order all medical tests. Most participants expected that their GP would make this decision and that the tests would be beneficial. These findings are aligned with previous research that patients overestimate the benefits and underestimate the harms of medical interventions. ${ }^{818}$ Implementation of the five Choosing Wisely questions into practice is complicated by patients' beliefs, attitudes, knowledge and emotions. ${ }^{25}$ Although the Choosing Wisely literature emphasises the need to change patients' expectations, ${ }^{418}$ few studies have examined implementation facilitators and barriers from patients' perspectives. The current study highlights the role of patients' motivation in using the five Choosing Wisely questions and de-implementation of low value care.

Our findings also indicate a lack of consumer and patient awareness of the five questions. This is reflective of the emphasis to date of the Choosing Wisely campaign on disinvestment by health practitioners and healthcare organisations in low value care, without similar efforts being made to educate patients and consumers about how to engage in shared decision-making by using the five questions.

Some participants commented on the value of the five Choosing Wisely questions as a signal that patients were permitted to ask questions of their doctor. The availability and application of communication tools such as the five Choosing Wisely questions may assist patients in addressing their lack of knowledge and the power imbalance between patients and clinicians. Although this may improve shared decision-making in de-implementation of low value care, support for GPs to engage and educate patients about using the five Choosing Wisely questions is needed. This may require a focus on giving patients permission to ask their doctors questions about the benefits of a watch and wait approach.

\section{Further research}

The five Choosing Wisely questions are a communication rather than a decision support tool. Research about decision support tools inclusive of communication guides and de-implementation in primary care contexts is warranted with focus on understanding patient motivation and also on empowering patients to ask questions and engage in shared decision-making. Research with larger representative samples to ascertain patients' perspectives would add knowledge at population levels. Further research is required in regard to the implementation of the 
five Choosing Wisely questions in defined populations with potentially different motivation for shared decision-making such as people living with chronic illness engaged in self-management.

\section{STUDY STRENGTHS AND LIMITATIONS}

Findings from the current study may have application to similar contexts of care elsewhere. The study included a small sample using semistructured interviews. This enabled a detailed descriptive exploration of participant's attitudes, beliefs and knowledge, and identification of factors and processes facilitating and constraining patients' use of the Choosing Wisely questions. The participants in the current study were all referred for and attended a CT scan and nothing is known about use of the five Choosing Wisely questions among patients who were not referred for a CT scan. Many participants had experienced a previous CT scan and this may have limited their perceived need to question their doctor. The timing of the interview after the CT scan may have predisposed participants to assume that the test was beneficial. Additionally, three interviews were conducted 1 week after their scan and this may have affected recall bias.

\section{CONCLUSIONS AND IMPLICATIONS FOR PRACTICE}

Shared decision-making is a principle underpinning Choosing Wisely. This ideal may not be matched in practice where patients seek a solution from an authoritative expert clinician such as a medical practitioner. Patients may require education that they are permitted to ask questions of their medical practitioner. Patients' motivation to engage in shared decision-making requires clinicians' understanding of patients' attitudes, beliefs, knowledge and emotions. Systems support and education for healthcare practitioners in effective communication is essential. However, this needs to emphasise communication with patients with varying motivation to engage in shared decision-making. Skilful application with patients of available communication tools, such as the five Choosing Wisely questions, and paid clinician time to undertake this important healthcare practice are imperative to future success in implementation of Choosing Wisely.

\section{Author affiliations}

${ }^{1}$ School of Nursing and Midwifery, Monash University, Clayton, Victoria, Australia ${ }^{2}$ Monash Health, Clayton, Victoria, Australia

${ }^{3}$ Imaging, Monash University, Clayton, Victoria, Australia

${ }^{4}$ Radiology, Monash Health, Clayton, Victoria, Australia

${ }^{5}$ Centre for Clinical Effectiveness, Monash Health, Clayton, Victoria, Australia

${ }^{6}$ Children's Cancer Foundation, Hudson Institute of Medical Research, Clayton, Victoria, Australia

${ }^{7}$ School of Nursing and Midwifery; Centre for Quality and Patient Safety Monash Health Partnership, Monash Health, Deakin University, Geelong, Victoria, Australia

Acknowledgements We are thankful for the support of all patients and carers who generously participated in the study. We acknowledge the support and time of staff at the medical imaging department at Casey Hospital including Nick Ardley, the reception staff and the nursing staff. We acknowledge the support by Monash Health and the Deakin University School of Nursing and Midwifery.

Contributors JA and AH designed and conceptualised the study with RK, SKG, AM, NN and AMH. JA recruited participants and collected data. JA and AH analysed data. $\mathrm{JA}$ and $\mathrm{AH}$ drafted the manuscript with support from RK, SKG, AM, NN and AMH.

Funding This work was supported by funding from Monash Health, Clayton Victoria, Australia.

Competing interests None declared.

Patient consent for publication Not required.

Ethics approval In accordance with the Australian National Health and Medical Research Council guidelines, the Ethics Committees at the healthcare organisation and Deakin University provided ethics approvals. Patients and carers were voluntary participants in the study. Following an explanation of the study, guided by the Participant Information and Consent Form, participants provided verbal and written consent. All data were de-identified. Project identifier numbers were allocated to all qualitative information.

Provenance and peer review Not commissioned; externally peer reviewed.

Data availability statement All data relevant to the study are included in the article or uploaded as supplementary information.

Open access This is an open access article distributed in accordance with the Creative Commons Attribution Non Commercial (CC BY-NC 4.0) license, which permits others to distribute, remix, adapt, build upon this work non-commercially, and license their derivative works on different terms, provided the original work is properly cited, appropriate credit is given, any changes made indicated, and the use is non-commercial. See: http://creativecommons.org/licenses/by-nc/4.0/.

\section{REFERENCES}

1. Wolfson D, Santa J, Slass L. Engaging physicians and consumers in conversations about treatment overuse and waste: a short history of the choosing wisely campaign. Acad Med 2014;89:990-5.

2. Greene SE, Massone R. A survey of emergency medicine residents' perspectives of the choosing wisely campaign. Philadelphia, Pennsylvania: Elsevier B.V, 2015: 853-5.

3. Zikmund-Fisher BJ, Kullgren JT, Fagerlin A, et al. Perceived barriers to implementing individual choosing wisely recommendations in two national surveys of primary care providers. J Gen Intern Med 2017;32:210-7.

4. Schlesinger M, Grob R, Treating GR. Treating, fast and slow: Americans' understanding of and responses to low-value care. Milbank Q 2017;95:70-116.

5. Levinson W, Kallewaard M, Bhatia RS, et al. 'Choosing wisely': a growing international campaign. BMJ Qual Saf 2015;24:167-74.

6. Stacey D, Légaré F, Lewis K, et al. Decision AIDS for people facing health treatment or screening decisions. Cochrane Database Syst Rev 2017;19.

7. Joseph-Williams N, Elwyn G, Edwards A. Knowledge is not power for patients: a systematic review and thematic synthesis of patientreported barriers and facilitators to shared decision making. Patient Educ Couns 2014;94:291-309.

8. Hoffmann T DMC. Patients' expectations of the benefits and harms of treatments, screening, and tests: a systematic review. Journal of the American Medical Association 2015;175:274-86.

9. Choosing Wisely. Choosing wisely: a special report on the first five years USA, 2019. Available: http://www.choosingwisely.org/wpcontent/uploads/2017/10/Choosing-Wisely-at-Five.pdf

10. Hahn C, Kavanagh B, Bhatnagar A, et al. Choosing wisely: the American Society for radiation oncology's top 5 list. Pract Radiat Oncol 2014;4:349-55.

11. Lusiani L, Frediani R, Nardi R, et al. Doing more does not mean doing better: the FADOI contribution to the slow medicine program for a sustainable and wise healthcare system. Italian Journal of Medicine 2015;9:281-6.

12. Simos $D$, Hutton $B$, Clemons $M$. Are physicians choosing wisely when imaging for distant metastases in women with operable breast cancer? J Oncol Pract 2015;11:62-8.

13. Figar S, Finakly S, Wong CJ, et al. Retrospective audit of plain film imaging in acute ankle trauma: are we choosing wisely? J Med Imaging Radiat Oncol 2017;61:149-50.

14. Hair H, Boudreau D, Rice C, et al. Mp18: a patient focused information design intervention to support the $\mathrm{mTBI}$ choosing wisely recommendation. CJEM 2017;19:S71. 
15. Lin MP, Nguyen T, Probst MA, et al. Emergency physician knowledge, attitudes, and behavior regarding ACEP's choosing wisely recommendations: a survey study. Acad Emerg Med 2017:24:668-75.

16. Gieniusz M, Kozikowski A, Williams M, et al. Peg tubes in patients with advanced dementia: Are we 'choosing wisely?'. J Am Geriatr Soc 2017;65:S77.

17. Silverstein W, Lass $\mathrm{E}$, Born $\mathrm{K}$, et al. A survey of primary care patients' readiness to engage in the de-adoption practices recommended by choosing wisely Canada. BMC Res Notes 2016;9:301-8.

18. MedicineWise NPS.Choosing wisely Australia 2017 report. Canberra Choosing Wisely Australia; 2017.

19. Clifford AM, Ryan J, Walsh C, et al. What information is used in treatment decision AIDS? A systematic review of the types of evidence populating health decision AIDS. BMC Med Inform Decis Mak 2017;17:1-15.

20. Pollard S, Bansback N, Bryan S. Physician attitudes toward shared decision making: a systematic review. Patient Educ Couns 2015;98:1046-57.

21. Elwyn G, Scholl I, Tietbohl C, et al. "Many miles to go ...": a systematic review of the implementation of patient decision support interventions into routine clinical practice. BMC Med Inform Decis Mak 2013;13.
22. O'Brien BC, Harris IB, Beckman TJ, et al. Standards for reporting qualitative research: a synthesis of recommendations. Acad Med 2014;89:1245-51.

23. Cane J, O'Connor D, Michie S. Validation of the theoretical domains framework for use in behaviour change and implementation research. Implement Sci 2012;7:37-53.

24. Michie S, Johnston M, Abraham C, et al. Making psychological theory useful for implementing evidence based practice: a consensus approach. Qual Saf Health Care 2005;14:26-33.

25. Atkins L, Francis J, Islam R, et al. A guide to using the theoretical domains framework of behaviour change to investigate implementation problems. Implementation Science 2017;12.

26. Spencer L, Ritchie J, Ormston R, et al. Anaysis principles and processes. In: Ritchie J, McNaughton Nicholls C, j L, et al, eds. Qualitative Research Practice: A Guide for Social Science Students \& Researchers. London: Sage, 2014: 269-95.

27. Spencer L, Ritchie J, O'Connor W, et al. Analysis in practice. In: Ritchie J, Lewis C, McNaughton Nicholls C, et al, eds. Qualitative Research Practice: A Guide for Social Science Students \& Researchers. London: Sage, 2014: 295-347.

28. Choosing Wisely Australia. 5 questions to ask your doctor or other healthcare provider, 2019. Available: http://www.choosingwisely.org. $\mathrm{au} /$ resources/consumers/5-questions-to-ask-your-doctor 